Research paper

\title{
Cognitive outcome in epileptic patients in a tertiary care centre in Kolkata, India
}

\author{
Gautam Das $^{\mathrm{a}, *}$, Piya Saha ${ }^{\mathrm{b}}$, Pritha Mukhopadhyay ${ }^{\mathrm{b}}$, Shankar P. Saha ${ }^{\mathrm{c}}$ \\ a Department of Medicine, North-24 Parganas District Hospital, Barasat, India \\ ${ }^{\mathrm{b}}$ Department of Psychology, University of Calcutta, 92, A.P.C. Road, Kolkata, India \\ ${ }^{\mathrm{c}}$ Department of Neurology, N.R.S. Medical College, 138, AJC Bose Road, Kolkata, India
}

\section{A R T I C L E I N F O}

\section{Article history:}

Received 4 February 2016

Accepted 26 April 2016

Available online 27 May 2016

\section{Keywords:}

Epileptic seizures

Idiopathic

Generalized tonic-clonic seizure

Complex partial seizure

Cognitive functions

\begin{abstract}
A B S T R A C T
Objective: The objective of the study is to assess the diverse cognitive dysfunctions in epileptic patients. Methods: Generalized tonic-clonic seizure (GTCS) and complex partial seizure (CPS) patients and those control matched and assessed for their IQ were undertaken for the present study. ANOVA test was used to assess the differences between GTCS and CPS with the control.

Results: GTCS and CPS exhibit lower scores than the control. In number cancellation task, they are at par. GTCS performed poorer than CPS.

Conclusion: Epileptic group showed impairment in working memory function, visuo-spatial skill, processing speed, visuo-perceptual attainment and reasoning ability. The deficit is more pervasive in GTCS group.

(c) 2016 Indian Epilepsy Society. Published by Elsevier, a division of Reed Elsevier India, Pvt. Ltd. All rights
\end{abstract} reserved.

\section{Introduction}

Cognitive behavioural problems were recognized in patients with epilepsy in ancient times and documented in the 19th century neurological literature. The most reported cognitive complaints in adults are mental slowness, memory impairment and attention deficits. Epileptic seizures can cause cognitive and neuropsychological alteration. Assessment of diverse cognitive functions is mandatory in epileptic patients to device holistic cognitive intervention programme, which is the ultimate aim of any clinical assessment. Cognitive impairment in epileptic patients has been attributed to interactions of genetic factors, different epilepsy syndromes, subclinical epileptiform discharges, psychosocial issues and treatment with antiepileptic drugs. ${ }^{1}$ Generalized tonic-clonic seizure (GTCS) and complex partial seizure (CPS) are two subgroups of idiopathic epilepsy (IGE). GTCS involves both cerebral hemispheres. The cognitive function of patients with GTCS tends to be somewhat lower than in the general population. ${ }^{2}$ Neuroimaging studies demonstrated that cortical and subcortical networks, including

Abbreviations: GTCS, generalized tonic-clonic seizure; CPS, complex partial seizure; HC, healthy control; DF, digit forward; DB, digit backward; AED, antiepileptic drugs.

* Corresponding author. Tel.: +91 9433145795.

E-mail address: drgautamdasmd@gmail.com (G. Das). regions of the fronto-parietal association cortex, thalamus, brainstem and cerebellum, are adversely affected in GTCS. ${ }^{3}$ Because no underlying lesion can be discerned in idiopathic epilepsies, IGE has been thought to be the best model for studying the relationship of epilepsy and cognition (Christopher Helmstaedter). A uniform pattern of cognitive impairment may not be predicted in epilepsy as it depends on the unique pathophysiology of underlying seizure disorder.

With this perspective, in the present study attempt has been taken to explore diverse cognitive functions in epileptic patients to identify the nature of cognitive dysfunctions in the specific diagnostic category of IGE.

\section{Materials and methods}

The study consists of two groups and was cross-sectional with case control design. Purposive sampling has been used in this study. After controlling IQ (i.e., those who fall in 25th to 50th percentile according to Standard Progressive Matrices), clinical sample $(n=28)$ of right-handed patients, with mean age of $22.7 \pm 4.35$ years, mediocre socio-economic status, generalized tonic-clonic seizure (GTCS) $(n=14)$, complex partial seizure of temporal lobe origin $(n=14)$, was taken from neurology outdoor of N.R.S. medical college and hospital, Kolkata, India. The diagnosis of epilepsy was made 
through detailed clinical examination and corroborated with EEG finding at times.

Patient with any traumatic brain injury, mentally challenged patients, any structural brain lesion as revealed by imaging and all secondary cases of epilepsy were excluded. Handedness, which is extremely relevant in terms of neuropsychological assessment, was determined with 10-item Edinburg Handedness Inventory. The circumstances of seizure's occurrences, their semiology, duration and evolution were noted. The medical context was systemically explored to list familial and personal antecedents, any psychiatric disorders and central nervous system drugs. Patient's activity of daily living was sought (ADL; Katz 1983). The neurological examination was done to assess motor function, saccades, Parkinsonian symptoms, any movement disorder, gait and sensory function. Brief interview was done to explore main biographical steps and milestones in personal and familial life. Various blood tests like sugar, electrolytes, liver function test (LFT) and thyroid profile were done to rule out any infective and metabolic causes. EEG was observed for interictal activities. Imaging was done to rule out any structural or vascular changes.

To minimize variations in AEDs' (anti-epileptic drugs) related factors on cognitive functions, we restricted the drug to sodium valproate, divalproex sodium, oxcarbazepine, lamotrigine and levetiracetam mainly for patients with epilepsy on regular treatment for at least the past 6 months, and we performed the serum levels of AEDs to choose patients with their serum levels within standard therapeutic range to exclude the effect of drug toxicity. This serum level measurement was restricted to valproate only, because that was the predominant drug used and also because that of the other drugs was not available. Other AEDs were added or substituted by valproate as permitted by the patient's clinical condition.

The sample of health participants recruited from community (HC) ( $n=23$, mean age $=25.9 \pm 5.48$ years $)$ matched with age, sex, education, handedness, socio-economic status and IQ was selected through snowball technique and screened through General Health Questionnaire. Participants scoring above the cut-off value of 4 were excluded. Exclusion criteria also included any psychiatric, neurological or sensory impairment.

Ethical committee permission was obtained for conducting the research. All the participants who gave their consent to participate in the study were corroborated in the study.

\subsection{Measures}

- Standard progressive matrices ${ }^{4}$

- Digit $\operatorname{span}^{5}$

- Digit symbol ${ }^{5}$

- Object assembly ${ }^{5}$

- Block design ${ }^{5}$

- Number cancellation 6

\subsection{Statistical analysis}

The data were tested for normative distribution using the Levene's test. Since most of the neuropsychological variables were normatively distributed, parametric analysis was carried out. Oneway analysis of variance (ANOVA) test was used followed by post hoc test to compare GTCS and CPS with their control counterparts. The critical value required for significance was set at 0.05 level. Statistical Package for Social Sciences (SPSS) version 15.0 was used for analyses and all reported $p$ values are two-tailed.

\section{Results}

The two groups were comparable in terms of age (mean age $=22.7 \pm 4.35$ years and $25.9 \pm 5.48$ years), sex and years of
Table 1

Socio-demographic description of the sample.

\begin{tabular}{lll}
\hline Category & $\begin{array}{l}\text { Clinical sample } \\
(n=28)\end{array}$ & $\begin{array}{l}\text { Healthy control }(\mathrm{HC}) \\
(n=23)\end{array}$ \\
\hline Age & $22.7 \pm 4.35$ years & $25.9 \pm 5.48$ years \\
Education & $11.1 \pm 2$ & $11.1 \pm 1.93$ \\
Age of onset & $14.7 \pm 6.8$ years & \\
Frequency & $2.6 \pm 1.06$ & \\
Duration & $8.6 \pm 6.67$ years & \\
\hline
\end{tabular}

education. In epilepsy group, duration of illness ranged from $8.6 \pm 6.67$ years, age of onset is between $14.7 \pm 6.8$ years and frequency ranged between $2.6 \pm 1.06$ years. The socio-demographic details of the two groups are provided in Table 1.

\subsection{Group differences among GTCS, CPS and HC}

Epileptic subgroups GTCS, CPS and HC were compared. Epileptic subgroups, GTCS and CPS, exhibit lower scores than HC in digit span task ( $F$ value-16.110*), in both digit forward ( $F$ value-24.791*) and backward tasks ( $F$ value-12.676*), digit symbol task ( $F$ value$\left.11.720^{*}\right)$, object assembly ( $F$ value-27.899*) and similarities task $(F$ value- $16.237^{*}$ ). In number cancellation, epilepsy subgroups are as per with HC ( $F$ value-0.680). In digit symbol and object assembly task, GTCS group $(2 \pm 1.1$ and $3.4 \pm 1.4)$ performed poorer as compared to CPS group ( $5 \pm 1.4$ and $5.4 \pm 2.3$ ). The findings are elaborated in Table 2.

\section{Discussion}

Neurocognitive impairment deserves investigation in epileptic patients, owing to the complex relationship between seizures and cognitive dysfunctions. The cognitive functions of attention, working memory, visuo-spatial and visuo-constructional abilities and psychomotor speed have been considered in this study, as these are the major domains to adversely affect brain-behaviour relationship.

A widespread neural network in the brain subserves the attention function. Digit forward (DF) taps the selectivity aspect of attention and is closely related to freedom from distractibility. In our study, the GTCS and CPS fared worse than the normal control in DF task, with equal vulnerability to distractibility indicating their deficit in attentional efficiency. It appears that impairment in attention network in IGE group is severe enough to adversely affect the DF task, which involves very minimal rehearsal process in phonological storage system of the working memory. Affection of the normal storage system of the working memory, involving prefronto-parietal and prefronto-temporal regions, is inferred in both the epileptic groups. Compromised selective attention in GTCS and CPS may not spare involvement of inferior parietal lobule in the left hemisphere, a crucial region for the functioning of the phonological short-term storage.

Table 2

Comparison of scores of GTCS, CPS and HC.

\begin{tabular}{lcccc}
\hline Test & $\begin{array}{l}\text { GTCS } \\
(n=14)\end{array}$ & $\begin{array}{l}\text { CPS } \\
(n=14)\end{array}$ & $\begin{array}{l}\text { Healthy } \\
(n=23)\end{array}$ & $F$ value \\
\hline Digit span & $4.7 \pm 1.6$ & $5.8 \pm 1.9$ & $8.2 \pm 2$ & $16.110^{*}$ \\
Digit forward & $6.3 \pm 1.6$ & $6.8 \pm 1.4$ & $9.1 \pm 1.02$ & $24.791^{*}$ \\
Digit backward & $4.4 \pm 3.1$ & $4.4 \pm 1.5$ & $7.3 \pm 1.3$ & $12.676^{*}$ \\
Digit symbol & $2 \pm 1.1$ & $5 \pm 1.4$ & $6 \pm 2.5$ & $11.720^{*}$ \\
Block design & $4 \pm 1.3$ & $4.8 \pm 1.4$ & $7.4 \pm 1.6$ & $27.899^{*}$ \\
Object assembly & $3.4 \pm 1.4$ & $5.4 \pm 2.3$ & $6.8 \pm 1.6$ & $15.458^{*}$ \\
Similarities & $3.1 \pm 1.3$ & $4.1 \pm 2.2$ & $6.7 \pm 2.1$ & $16.237^{*}$ \\
Number cancellation & $151.4 \pm 10.9$ & $154 \pm 6.7$ & $154.5 \pm 6.9$ & 0.680 \\
\hline
\end{tabular}

Significant at 0.05 level. 
Digit backward (DB) performance is also equally compromised in both GTCS and CPS as compared to matched healthy controls (HC). Poor performance of epileptic groups on DB task, which imposes high working memory load with demand for executive control, indicates their difficulty in exercising adequate executive control over thought processes. Lesion and functional imaging studies revealed that the neural correlates of working memory and phonological store depend largely upon the left inferior parietal cortex and the rehearsal process depends on the left inferior frontal gyrus (IFG) (typically described as Broca's area), premotor area and supplementary motor area. ${ }^{8}$ The central executive system relies heavily particularly on the dorsolateral prefrontal cortex (DLPFC) and the dorsal anterior cingulate cortex (ACC). Dysexecution in epilepsy groups may be owing to impaired functional connectivity between these areas, as is understood from our findings.

Average performance of epileptic group in number cancellation task requires explanation. Number cancellation task measures the capacity for selective attention, concentration, visual scanning, rapid response activation and inhibition, which predominantly utilizes fronto-parietal network, mediated by a dorsal system, including the superior parietal lobule and the superior portion of the dorsal prefrontal cortex. Though both the digit repetition and number cancellation have demand on attention function, epileptic patients' average performance on the latter indicates perhaps that the epileptic group does not face difficulty to work on number cancellation task as the stimulus material has been presented in their visual field, with least or no demand on retrieval process; but retrieval from phonological storage in digit repetition task demands left hemispheric participation. Heilman ${ }^{10}$ has argued that right hemisphere plays a greater role for attention process, which corroborates with PET study outcome that explained that the system activated by external stimuli is lateralized to the right hemisphere, particularly in right handed individuals. As the right hemisphere provides alerting mechanisms for both sides of the body, attentional impairment is more pervasive with right-sided hemisphere lesions. ${ }^{10}$ Thus no group difference indicates that the right hemispheric function of epileptic subgroups is more or less at par with the HC.

Visuo-spatial task of block design has a demand on the higher cognition of visuo-spatial concept attainment, organizing abilities, processing speed and the ability to separate figure ground in visual stimuli, which is associated with predominantly right hemisphere functions, particularly parietal region. ${ }^{11}$ Below average performance of epileptic group in this task denotes that the demand of the aforesaid task could not be satisfied by both the epileptic groups. It suggests that the right parietal region, which is responsible for visuo-spatial concept attainment, has not been spared by the neuropathology caused by either type of epilepsy. Also in object assembly task, both the epileptic subgroups scored significantly poor than the healthy participants. The object assembly task has a demand for understanding the gestalt from the disjointed parts of the object when no model for comparison is provided. This function is vulnerable to posterior lesions, and more so to the right than left hemisphere lesions. ${ }^{12}$ Since the stimulus materials are to be perceived as parts of the whole and the whole object is to be constructed out of parts, with no given model in the perceptual field, it becomes a critical situation for the epileptic groups to assemble the objects, signifying their incapacity to form an image of the object that perhaps has made the task difficult for them. Both object assembly task and block design task are vulnerable to posterior lesions but GTCS performed poor compared to CPS in object assembly task, signifying that the neural networks for these two tasks may be dissociable.

Worse performance of GTCS compared to CPS suggests that since the object assembly requires synchronous function of the whole brain, the impairment of organizational ability in task utilizing neural network underlying visuo-constructional ability could be more severe in GTCS that that which affects the whole cortex, in contrast to CPS, where the abnormal electrical discharge is confined to temporal regions. The finding gives an impression that specific part of temporal areas, if it has any role in this task, has been spared for the object assembly task in CPS.

Both GTCS and CPS groups showed deficit in digit symbol subtest as compared to HC. The test requires attention, visuoperceptual processing, working memory and psychomotor speed. Poor performance of the epileptic group in psychomotor speed has been reported by others. ${ }^{14}$ Greater impairment in digit symbol and object assembly tasks in GTCS may be attributable to more diffused frontal-subcortical circuitry in GTCS than the focal seizures in CPS. ${ }^{15}$ The same whole brain hypothesis for object assembly holds for digit symbol task to explain worse performance of GTCS than the CPS. Visuo-perceptual processing, working memory and psychomotor speed are more compromised in GTCS than CPS, as the lesions are more pervasive in the former group.

Similarities' subtest measures verbal concept formation and reasoning. The performance of epileptic groups was poor as compared to HC, signifying that epileptic subgroups form concept at concrete level ${ }^{16}$ owing to difficulty in categorisation. However, antiepileptic drug (AEDs) treatment can have positive, as well as negative effects on cognition. Antiepileptic drugs can synergistically exacerbate the deficits arising from the neurological pathophysiology by suppression of neuronal excitability or the enhancement of inhibitory neurotransmission in certain brain areas. Findings of other researchers suggest a reconsideration of the negative impact of interictal epileptic dysfunction on cognition. ${ }^{17}$ The pattern of cognitive impairment, however, depends largely on the pathophysiology underlying the seizure disorder.

\section{Conclusion}

Impairment in cognitive functions is evident in both the epilepsy subgroups with equal standing in all the cognitive functions, where they fared worse than healthy participants. However, greater impairment in digit symbol and object assembly tasks in GTCS may be explicable in terms of their diffuse frontalsubcortical circuitry, while in CPS, the epicentre is restricted to a given region. However, in CPS of temporal origin, the non-temporal effect may be a distal effect. There is converging evidence that the so-called older AEDs (Phenobarbital, phenytoin, carbamazepine and valproate) are more problematic with respect to cognition and behaviour than so-called newer AEDs (lamotrigine, gabapentine, levitiracetam, etc.). In our study, the drug was restricted to sodium valproate and divalproex sodium for treatment to minimize the adverse effect of drug on cognition.

\section{Limitation of our study}

In order to match all three groups in intelligence, the sample size has been reduced to 28 in totality from the initial pool of 100. Initially, from 100 patients, after excluding metabolic, infective causes and associated motor, sensory and other neurological problems, it came down to 56. Then, on matching for IQ, it reduced to 28 . Thus, generalization of the present finding is warranted. The study-time period was only 1 year, which may not be sufficient to gauge the cognitive decline without a longer follow-up line. Indeed, many of the patients came to our clinic with multiple drugs along with valproate. We tried to minimize the drug as far as practicable according to the clinical situation. Finally, we did stick to valproate as the predominant drug. 


\section{Conflicts of interest}

The authors have none to declare.

\section{Acknowledgements}

Professor Pritha Mukhopadhyay, Coordinator, CPEPA.

This research was supported by University Grants Commission (UGC) sponsored research scheme "Centre with Potential for Excellence in Particular Area (CPEPA)" at University of Calcutta [Scheme Ref. F.R.8-2/2008].

\section{References}

1. van Rijckevorsel K. Cognitive problems related to epilepsy syndromes, especially malignant epilepsies. Seizure. 2006;15:227-234.

2. MacAllister WS, Schaffer SG. Neuropsychological deficits in childhood epilepsy syndromes. Neuropsychol Rev. 2007:17:427-444.

3. Henkin Y, Sadeh M, Kivity S, Shabtai E, Kishon-Rabin L, Gadoth N. Cognitive function in idiopathic generalized epilepsy of childhood. Dev Med Child Neurol. 2005;47:126-132.
4. Blumenfeld $\mathrm{H}$, Westerveld $\mathrm{M}$, Ostroff $\mathrm{RB}$, et al. Selective frontal, parietal and temporal networks in generalized seizures. Neurolmage. 2003:19:1556-1566.

5. Raven J, Raven JC, Court JH. Manual for Raven's Progressive Matrices E Vocabulary scales. Section 1: General overview. San Antonio, TX: Hercourt Assessment; 2003.

6. David W. The Measurement of Adult Intelligence: Wechsler Adult Intelligence Scale. 3rd ed. Baltimore, MD: Williams \& Witkins; 1979.

7. Rao SL, Subbakrishna DK, Gopukumar K. NIMHANS Neuropsychology Battery. 1st ed. Bangalore: National Institute of Mental Health and Neurosciences; 2004.

8. Vallar G, Papagno C. Neuropsychological impairments of short-term memory. In: Baddeley AD, Wilson BA, Watts FN, eds. In: Handbook of Memory Disorders. West Sussex, England: John Wiley \& Sons; 1995:135-165.

10. Heilman KM. Neuropsychology Clinical Approach. 4th ed. Walsh \& Darby: Elsevier Health Sciences; 1982.

11. Lezak M. Neuropsychological Assessment. 3rd ed. New York: Oxford University Press; 1995.

12. Newcombe F. Missile Wounds of Brain. Oxford: Oxford University Press; 1969.

14. Hermann B, Seidenberg M, Dow C, et al. Cognitive progress in chronic temporal lobe epilepsy. Ann Neurol. 2006;60:80-87.

15. Dodrill C, Robert W. Neuropsychological correlates of the electroencephalogram in epileptics. II. The waking posterior rhythm and its interaction with epileptiform activity. Epilepsia. 1976;17:101-109.

16. Hanfmann. Neuropsychology: A Clinical Approach. 4th ed. Walsh \& Darby: Elsevier Health Sciences; 1944.

17. Greener M. Beyond seizures: understanding cognitive deficits in epilepsy. Prog Neurol Psychiatry. 2013;17:31-32. 